\title{
Metastatic peritoneal sarcomatosis from radiation-induced osteosarcoma in a patient with previously treated pelvic Ewing's sarcoma: Report of a case
}

\author{
Rajendra Kumar $^{1 *}$, Romil Y. Patel ${ }^{1 \#}$, John E. Madewell ${ }^{1}$, Tariq J. Al-Zaid ${ }^{2}$, Jeanne M. Meis ${ }^{2}$ \\ ${ }^{1}$ Department of Diagnostic Imaging, The University of Texas MD Anderson Cancer Center, Houston, USA \\ ${ }^{2}$ Department of Pathology, The University of Texas MD Anderson Cancer Center, Houston, USA \\ Email: rajkumar@mdanderson.org, dr.rypatel@gmail.com, jmadewell@mdanderson.org, tariqjz1@yahoo.com, \\ jmmeis@mdanderson.org
}

Received 5 February 2013; revised 10 March 2013; accepted 3 April 2013

Copyright (c) 2013 Rajendra Kumar et al. This is an open access article distributed under the Creative Commons Attribution License, which permits unrestricted use, distribution, and reproduction in any medium, provided the original work is properly cited.

\begin{abstract}
Metastatic peritoneal sarcomatosis most commonly occurs from primary soft tissue sarcomas arising either within the abdomen or extremities. Metastatic peritoneal sarcomatosis from an osteosarcoma is extremely rare; only six cases have previously been reported. We report the first case of metastatic peritoneal sarcomatosis originating from radiation-induced osteoblastic osteosarcoma in a 22-year-old woman who had previously been treated for pelvic Ewing's sarcoma. Abdominal computed tomography, bone scintigraphy and FDG PET/CT demonstrated extensive finely nodular disseminated peritoneal lesions. Histopathologic examination of these peritoneal lesions revealed osteosarcomatosis. In summary, we describe an unusual case of metastatic peritoneal sar comatosis from secondary osteosarcoma arising in a previously irradiated pelvic Ewing's sarcoma.
\end{abstract}

Keywords: Peritoneal Sarcomatosis; Peritoneal Osteosarcomatosis; Radiation-Induced Osteosarcoma; Radiation-Induced Bone Sarcoma

\section{INTRODUCTION}

Metastatic peritoneal sarcomatosis is an unusual manifestation of primary sarcoma, in which the tumor disseminates into the peritoneal cavity [1]. It is usually associated with soft tissue sarcomas of the abdomen, such as abdominal gastrointestinal stromal tumors, liposarcomas and leiomyosarcomas, and infrequently, with soft

*Corresponding author.

\#Present address: Department of Radiology, Lake Medical Imaging and Vascular Institute, 801 E Dixie Ave., Ste. 104, Leesburg, Florida, USA. tissue sarcomas of the extremities [1,2].

Metastatic peritoneal sarcomatosis originating from a bone sarcoma, specifically osteosarcoma, is extremely rare. Only five cases of peritoneal sarcomatosis from primary osteosarcoma of bone have been reported so far [3]. There has also been a report of peritoneal sarcomatosis in a woman with primary osteosarcoma of uterus [4].

We report a woman with pelvic Ewing's sarcoma who was treated successfully with chemo-radiation. Seven years later, the patient developed secondary osteosarcoma in the irradiated pelvis, and subsequently, disseminated peritoneal sarcomatosis. This is the first reported case of peritoneal sarcomatosis arising from secondary osteosarcoma of bone.

\section{CASE REPORT}

A 22-year-old woman with Ewing's sarcoma of her right iliac bone was treated with chemo-radiation at an outside institution. She received a total radiation dose of $55.8 \mathrm{~Gy}$ to the right hemipelvis. She had complete remission following the treatment. However, seven year later she started having gradually increasing right hip pain. MRI of the pelvis revealed radio-osteonecrosis in the right hip, for which she underwent right total hip arthroplasty. Approximately 1 year later, when the right hip pain recurred, radiographs showed osteolysis along the acetabular component of the right hip prosthesis, suggesting osteomyelitis. However, microbiological stains and cultures from the hip joint aspirate were negative. Subsequently, at the time of revised right hip arthroplasty, biopsy of the right iliac bone from the site of the osteolysis revealed osteoblastic osteosarcoma (Figure 1).

The patient was referred to our institution for further treatment. At that time, pathologic review of the initial 


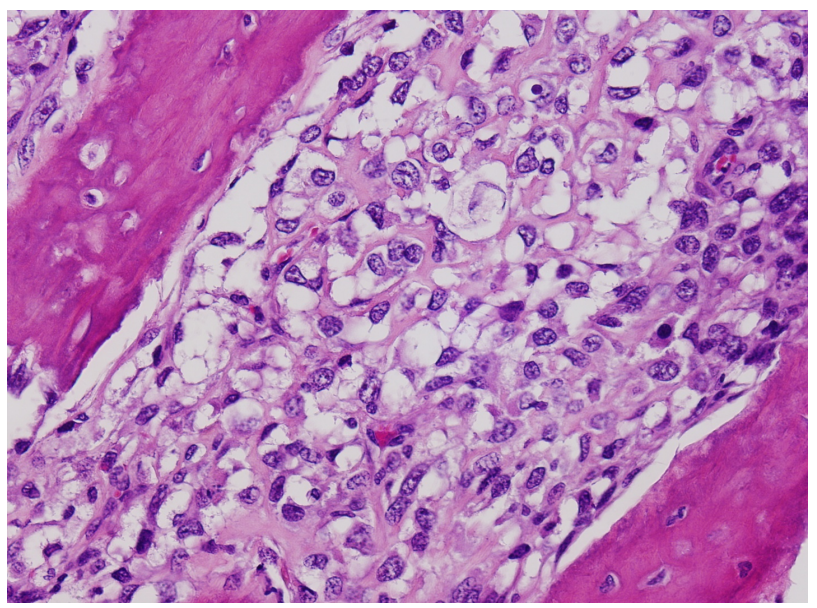

Figure 1. Tumor consists of an atypical round to oval cell proliferation with lace-like osteoid deposition, characteristic of osteosarcoma. (H\&E stain $\times 200)$.

pelvic tumor diagnosed as Ewing sarcoma was confirmed immunohistochemically, and with fluorescent in situ hybridization (FISH) studies that revealed gene rearrangement of the EWSR1 (22q12) locus. A technetium-99m MDP bone scan showed intense radiotracer uptake throughout the right hemipelvis and along the right hip (Figure 2(a)). An F-18 FDG PET/CT scan demonstrated a destructive bone tumor in the right hemipelvis with the tumor extension into the adjacent soft tissues (Figures 2(b) and (c)).

Following chemotherapy, the patient underwent right external hemipelvectomy and right hip disarticula tion. Approximately one month after the surgery, she developed ascites. Technetium-99m MDP bone scan at this time demonstrated foci of intense radiotracer uptake throughout the abdomen and pelvis (Figure 3(a)). Abdominal CT without contrast revealed diffuse finely calcified peritoneal metastases (Figure 3(b)). Following intravenous contrast, abdominal CT demonstrated enhancing finely nodular peritoneal thickening throughout the abdomen, suggesting peritoneal sarcomatosis (Figure 3(c)).

Ultrasound-guided peritoneal biopsy was performed. Histopathology confirmed peritoneal osteosarcomatosis (Figure 4).

The patient expired about one month later.

\section{DISCUSSION}

Post-radiation sarcomas account for between $0.5 \%$ and $5.5 \%$ of all sarcomas [5]. The incidence is increasing as cancer patients treated with chemotherapy are now living long enough to experience the oncogenic effects of radiation. Chemotherapy has also been shown to increase the relative risk of sarcoma development by a factor of 4.7 to 9 [5]. Bone sarcoma in a previously irradiated bone is well documented with a relative risk of 24.9 cases/10,000 person years after doses of 48 - 59.9 Gy, and 130 cases/10,000 person-years for patients receiving a radiation dose of $60 \mathrm{~Gy}$ or more [6,7]. No case of secondary sarcoma in children with Ewing sarcoma occurred with radiation dose of $<48 \mathrm{~Gy}$ [6]. The mean radiation dose associated with development of post-radiation bone sarcomas is $37 \mathrm{~Gy}$ (range, 9 - $132 \mathrm{~Gy}$ ) [5]. Radiation doses greater than 50 Gy cause complete destruction of the irradiated bone tumor tissue. However, the lower radiation dose of between 30 - 50 Gy at the periphery of the tumor may damage the cell reparative mechanisms, and induce genetic mutations within the tumor cells. Thus, it is the edge of the radiation field which is at enhanced risk for development of local secondary malignancy in a previously irradiated bone [5].

The estimated risk of developing secondary mali-
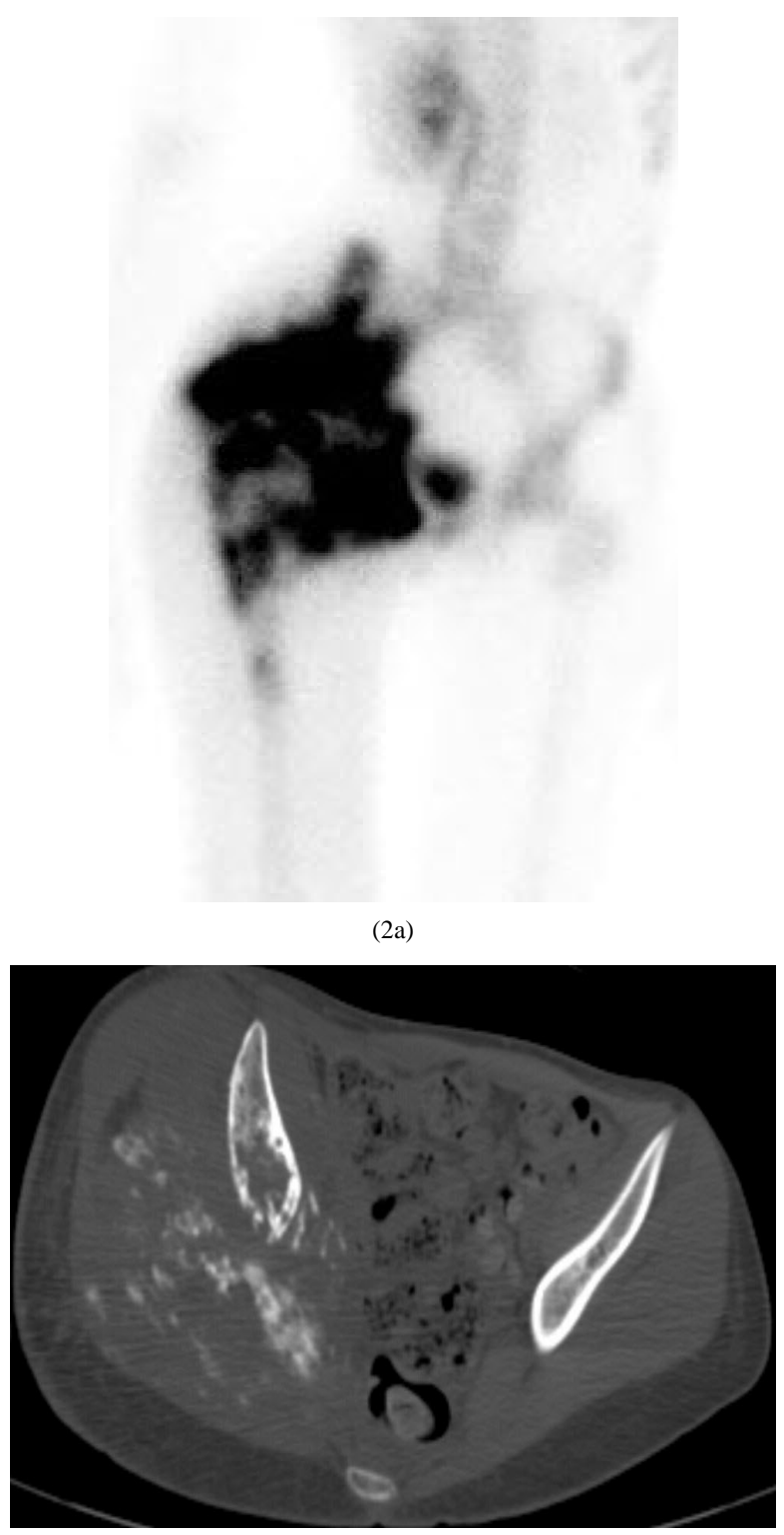

(2b) 


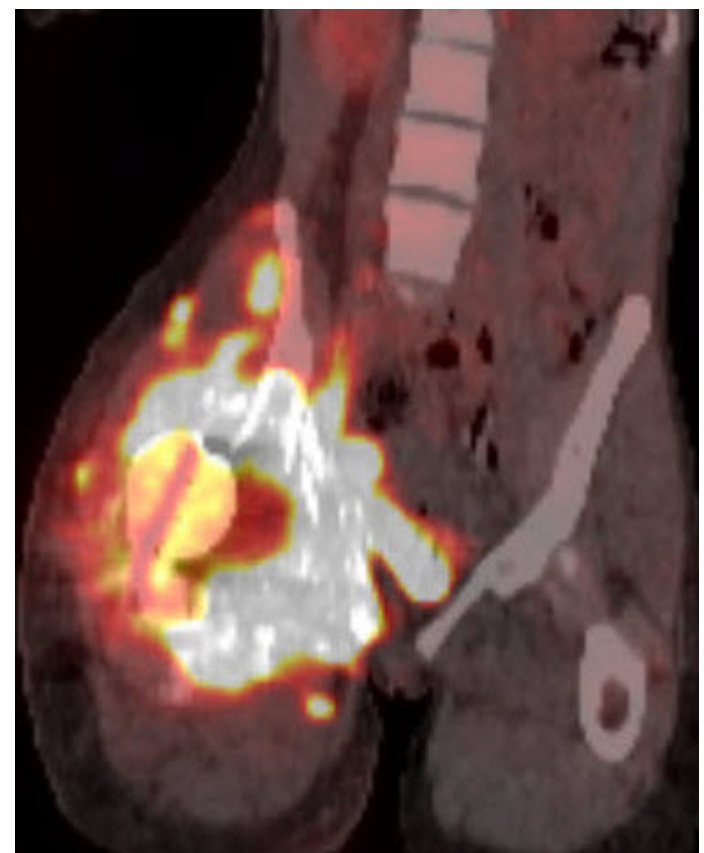

(2c)

Figure 2. (a) Technetium-99m bone scan demonstrates intense radiotracer uptake in right hemipelvis and proximal femur corresponding with radiation-induced osteosarcoma. No radiotracer uptake elsewhere in abdomen is present. (b) Unfused axial CT from F-18 PET/CT scan demonstrates destructive bony tumor with large partially mineralized extra-osseous soft tissue component in right hemipelvis. (c) Coronal fused F-18 FDG PET/CT image shows tumor is intensely hypermetabolic.

gnancy following radiation therapy for Ewing's sarcoma has been estimated to range from $2 \%$ - $10 \%$ [8]. Other studies corroborate this, with an estimated excess risk of radiation-induced osteosarcoma as secondary malignancy following primary Ewing's sarcoma treatment in 4.95 cases per 10,000 patients [6]. These findings have implications for managing primary pediatric neoplasms with radiotherapy (8). The mean overall 5 year survival figure for radiation-induced osteosarcoma is $40.2 \%$ [7].

The mean latent period between radiation exposure and subsequent bone malignancy varies between 4 and 17 years (range, 2.75 - 55 years) [5]. The latency period may depend on the age at the time of radiotherapy and radiation dose. The younger the patient and larger the radiation dose, the shorter is the latency period for secondary neoplasm to develop $[5,6]$.

The pelvis is a favorite site, and osteosarcoma is the most common secondary bone malignancy associated with radiation treatment, and prognosis for most post-radiation osteosarcomas is poor [5,7]. We believe the large radiation dose of $55.8 \mathrm{~Gy}$ for treatment of the pelvic Ewing sarcoma placed our patient at higher risk for development of a radiation-induced bone sarcoma, which occurred 7 years after the radiation treatment.

Metastatic peritoneal sarcomatosis from a primary soft tissue sarcoma is a known albeit infrequent event [1-3]. The most common tumors responsible for peritoneal sarcomatosis are retroperitoneal soft tissue sarcomas, such as gastrointestinal stromal tumors, liposarcomas and leiomyosarcomas, through direct spread [1-3]. Less often, extremity soft tissue sarcomas can also metastasize to the peritoneum, probably through hematogenous spread [2].

Five patients with primary osteosarcoma of bone who developed peritoneal metastases in the course of their disease have previously been reported [3]. These patients, varying in age from 14 years to 65 years, were all initially treated successfully with chemotherapy for the

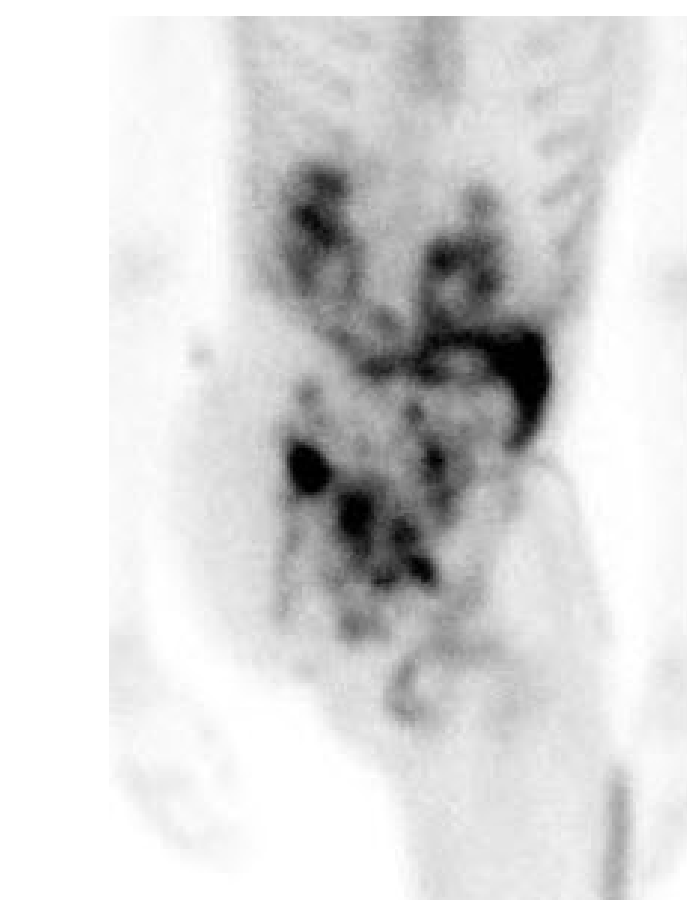

(3a)

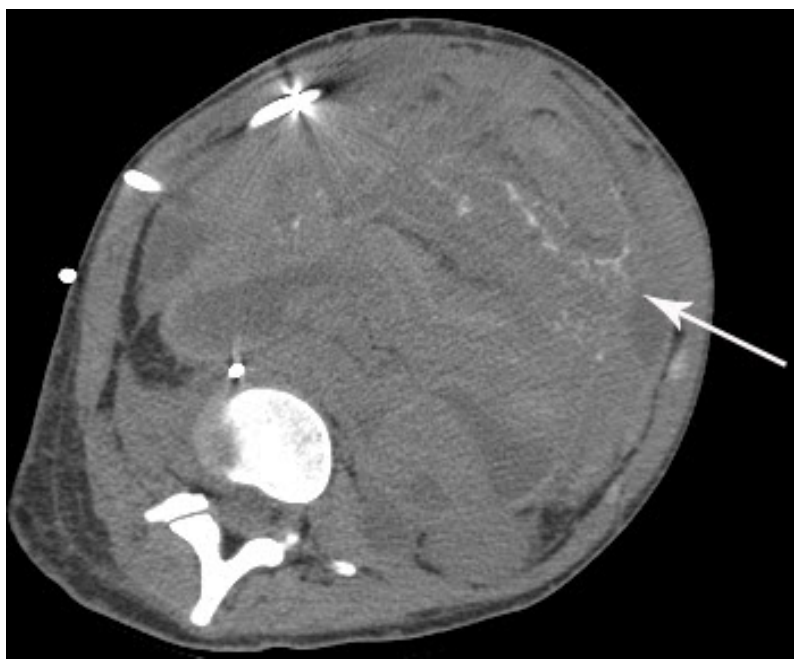

(3b) 


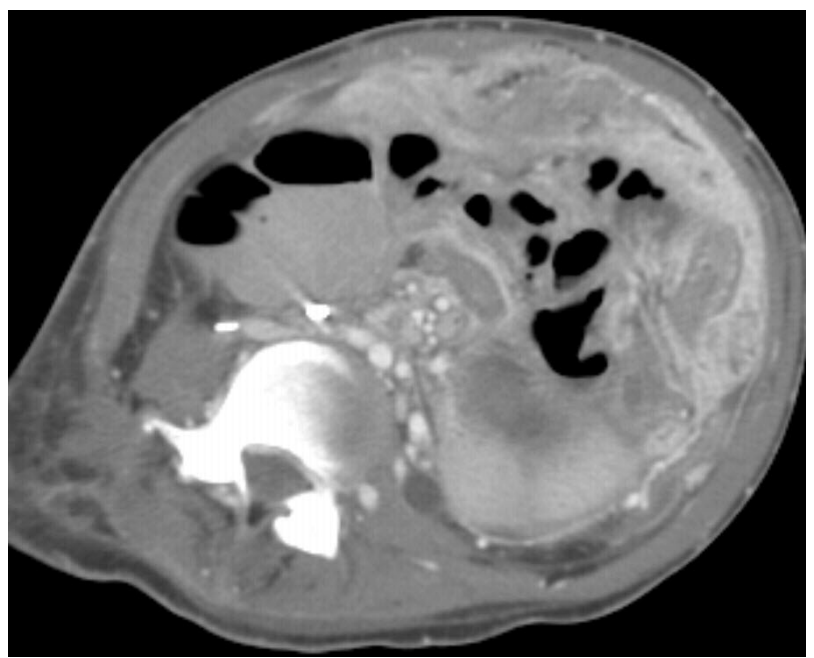

(3c)

Figure 3. (a) Technetium-99m bone scan shows intense radiotracer uptake throughout abdomen and pelvis due to peritoneal sarcomatosis. Also note activity in urinary collecting structures, right hemipelvectomy and right hip disarticulation. (b) Axial non-contrast CT of abdomen demonstrates diffuse mineralized fine nodular peritoneal thickening representing osteosarcomatosis (white arrow). (c) Contrast-enhanced axial CT of abdomen demonstrates enhancing diffuse finely nodularperitoneal thickening and ascites.

primary bone tumor. The time elapsed following successful treatment of the primary bone tumor and peritoneal involvement was between 19 months to 50 months. All the five patients with peritoneal osteosarcomatosis complained of gradually increasing abdominal pain. Two of them had ascites, while another patient with hepatic metastases and intestinal obstruction developed hemorrhagic ascites. Three patients had distinct calcified large metastatic peritoneal tumors, while the other two had disseminated small metastatic peritoneal nodules, similar to our case. Many of these metastatic nodules were mineralized. In two of the five cases, the metastatic peritoneal spread was believed to be hematogenous. In the remaining three cases, direct spread of the pulmonary metastases into the liver and peritoneum was suspected [3].

Direct peritoneal involvement with ascites was also observed in a woman with primary uterine osteosarcoma [4].

We believe the radiation-induced pelvic osteosarcoma in our patient also had direct tumor spread to the peritoneum, possibly facilitated by the pelvic surgery.

Prior to the era of chemotherapy, patients with primary osteosarcoma developed mostly lung metastases, but rarely had extrapulmonary metastases $[3,9]$. The extrapulmonary metastases found at autopsy in many of these patients were not evident prior to death [2,9]. In contrast, patients now treated with chemotherapy with longer disease-free survival develop fewer or at times, no

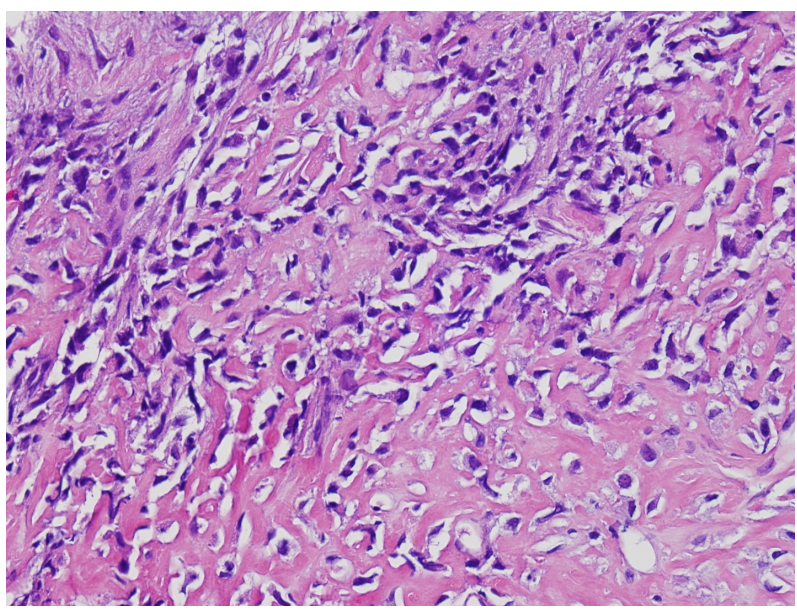

Figure 4. Peritoneal tumor consists of primitive malignant cell and osteoid (H\&E stain $\times 200)$.

pulmonary metastases, yet they tend have more extrapulmonary metastases later in the course of their disease. The reason behind this observation could be due to temporary suppression of some of the tumor cells, and elimination of other, more rapidly dividing tumor cells, with chemotherapy, while less sensitive tumor cells may persist, grow slowly, and manifest later as extrapulmonary metastases. Alternatively, pulmonary metastases may be more sensitive to intravenous chemotherapy [9]. In four of the five previously reported cases of primary bone osteosarcoma with peritoneal metastases, the first tumor recurrence was in the lungs [3], while the woman with primary osteosarcoma of the uterus with direct tumor spread to the peritoneum had no lung metastases [4]. Our patient with radiation-induced secondary osteosarcoma also developed direct peritoneal metastases without ever having lung metastases.

A radiation-induced sarcoma in a previously irradiated bone should be suspected, when after at least a 5-year disease-free period, new localized symptoms or signs of the tumor, especially in absence of local trauma or infection, recur. Presence of a soft tissue mass (probability, $100 \%$ ), particularly in combination with either bone destruction (95\%) or tumor matrix mineralization (53\%) on radiographs and CT is strongly suggestive of recurrent osteosarcoma [5].

Radiological diagnosis of metastatic peritoneal sarcomatosis in a patient with osteosarcoma can mimic more common metastatic peritoneal carcinomatosis and primary peritoneal tumors (1). Peritoneal sarcomatosis can manifest as a rather large, hypervascular peritoneal mass with variable ascites or hemoperitoneum, as reported in three of the previously reported cases [1,3]. Alternately, the metastases may appear as numerous small nodules disseminated throughout peritoneum. In the proper clinical setting, sudden onset of persistent abdominal pain or 
symptoms pertaining to intestinal obstruction should suggest metastatic peritoneal involvement [3].

Radiographs may demonstrate a partially calcified intraabdominal metastatic soft tissue mass or peritoneal metastases may appear as numerous small calcific densities scattered in the abdomen. Presence of mineralization within these metastases tends to favor osteosarcomatosis. A radionuclide bone scan is an effective diagnostic procedure for detection of mineralized peritoneal metastases $[10,11]$ (Figure 3(a)). Most small soft tissue metastases are poorly detected with radionuclide bone scan, but occasionally scintigraphy may reveal the first evidence of calcific metastatic disease [10]. Both abdominal CT (with or without contrast) and F-18 FDG PET/CT are highly sensitive in the detection of peritoneal and concurrent intra-abdominal metastases as well as the extent of peritoneal involvement (Figure 3). PET will also show metabolic activity of these metastatic lesions, while CT can detect both calcific metastatic peritoneal nodules and ascites [1]. MRI with intravenous Gadolinium can also show with advantage the size and extent of enhancing peritoneal metastases; however, abdominal CT is preferred $[1,2,5,12]$.

In all cases, clinical data, diagnostic imaging and histological examination are required to determine tumor extent, and establish diagnosis. Cyto-spun fluid examination should be performed when ascites is present [3].

Treatment of metastatic peritoneal osteosarcomatosis is uncertain because of the extreme rarity of these cases $[3,11]$. The standard treatment for metastatic peritoneal sarcomatosis from abdominal and extremity sarcomas is systemic chemotherapy and local radiotherapy. Focal metastatic peritoneal tumors can be surgically resected. Overall prognosis has been dismal; most patients died within a year [3].

In summary, we have described an unusual case of peritoneal metastases fromradiation-induced osteosarcoma in a patient previously irradiatedfor pelvic Ewing sarcoma.

\section{REFERENCES}

[1] Oei, T.N., Jagannathan, J.P., Ramaiya, N. and Ros, P.R. (2010) Peritoneal sarcomatosis versus peritoneal carcinomatosis: Imaging findings at MDCT. AJR American Journal of Roentgenology, 195, W229-W235. doi:10.2214/AJR.09.3907
[2] Zanarini, D. and Sugarbaker, P.H. (1997) Extremity soft tissue sarcoma with metastases to abdominopelvic surfaces. Journal of Surgical Oncology, 64, 68-72. doi:10.1002/(SICI)1096-9098(199701)64:1<68::AID-JS O13>3.0.CO;2-S

[3] Yeh, S.N. and Lu, M.Y. (2005) Peritoneal metastasis in osteosarcoma: Report of one case. Acta Paediatrica Taiwanica, 46, 230-234.

[4] Lin, J.-W., Ko, S.-F., Ng, S.-H., Eng, H.-L, Changchien, C.-C. and Huang, C.-C. (2002) Primary osteosarcoma of the uterus with peritoneal osteosarcomatosis. British Journal of Radiology, 75, 772-774.

[5] Sheppard, D.G. and Libshitz, H.I. (2001) Post-radiation Sarcomas: A review of the clinical and imaging features in 63 cases. Clinical Radiology, 56, 22-29. doi:10.1053/crad.2000.0599

[6] Kuttesch Jr., J.F., Wexler, L.H., Marcus, R.B., Fairclough, D., Weaver-McClure, L., White, M., Mao, L., Delaney, T.F., Pratt, C.B., Horowitz, M.E. and Kun, L.E. (1996) Second malignancies after Ewing's sarcoma: Radiation dose-dependency of secondary sarcomas. Journal of Clinical Oncology, 14, 2818-2825.

[7] Koshy, M., Paulino, A.C., Mai, W.Y. and Teh, B.S. (2005) Radiation-induced osteosarcoma in the pediatric population. International Journal of Radiation Oncology \& Biology \& Physics, 63, 1169-1174. doi:10.1016/j.ijrobp.2005.04.008

[8] Laskar, S., Mallick, I., Gupta, T. and Muckaden, M.A. (2008) Post-operative radiotherapy for Ewing sarcoma: When, how and how much? Pediatric Blood \& Cancer, 51, 575-580. doi:10.1002/pbc.21657

[9] Giuliano, A.E., Feig, S. and Eilber, F.R. (1984) Changing metastatic patterns of osteosarcoma. Cancer, 54, 21602164.

doi:10.1002/1097-0142(19841115)54:10<2160::AID-CN CR2820541016>3.0.CO;2-P

[10] Heinle, A., Carrasquillo, J.A. and Neumann, R.D. (1989) Two unusual cases of metastatic osteosarcoma detected by Tc-99m MDP bone scintigraphy. Clinical Nuclear Medicine, 14, 889-893. doi:10.1097/00003072-198912000-00006

[11] Westra, A., Schrijvers, D., Somville, J., Van Schil, P., Hubens, G. and Van Oosterom, A. (1998) Late peritoneal metastasis in a patient with osteosarcoma. Annals of Oncology, 9, 907-911. doi:10.1023/A:1008260216130

[12] Rossi, C.R., Casali, P., Kusamura, S., Baratti, D. and Deraco, M. (2008) The consensus statement on the locoregional treatmentof abdominal sarcomatosis. Journal of Surgical Oncology, 98, 291-294. 